\title{
Controlled dephasing of Andreev states in superconducting quantum point contacts
}

\author{
M. A. Despósito ${ }^{1}$ and A. Levy Yeyati ${ }^{2}$ \\ ${ }^{1}$ Departamento de Física, Facultad de Ciencias Exactas y Naturales, Universidad de Buenos Aires, 1428 Buenos Aires, Argentina \\ ${ }^{2}$ Departamento de Física de la Materia Condensada C-V. Facultad de Ciencias, Universidad Autónoma de Madrid, \\ E-28049 Madrid, Spain
}

(Received 28 March 2001; published 21 September 2001)

\begin{abstract}
We have studied the relaxation and dephasing processes in a superconducting quantum point contact induced by the interaction with an electromagnetic environment. Based on a density matrix approach we obtain the rates for the dissipative dynamics as function of the transmission, the phase difference on the contact, and the external impedance. Our calculation allows us to determine the appropriate range of parameters for the observation of coherent oscillations in the current through the contact.
\end{abstract}

DOI: 10.1103/PhysRevB.64.140511

PACS number(s): 74.80.Fp, 73.63.-b, 73.63.Rt

In a superconducting quantum-point contact (SQPC) the zero-voltage transport properties are determined by the socalled Andreev states within the superconducting energy gap $\Delta$, which are given by ${ }^{1}$

$$
E_{ \pm}(\phi)= \pm \Delta \sqrt{1-\tau \sin ^{2}(\phi / 2)},
$$

where $\phi$ is the phase difference between the superconducting electrodes and $\tau$ is the normal transmission coefficient for each conduction channel. Atomic contacts, produced by scanning tunneling microscope or the mechanically controllable break-junction (MCBJ) technique, have provided an almost ideal realization of an SQPC with a few conduction channels whose transmissions can be determined experimentally. ${ }^{2}$ Different methods for Andreev level spectroscopy have been suggested. ${ }^{3}$

The Andreev states in a single channel SQPC constitute an interesting realization of a two-level system with well characterized parameters, which could be proposed as a solid state realization of a quantum qubit. ${ }^{4}$ The phase difference through the contact can be fixed by the magnetic flux in a superconducting ring geometry like the one depicted in Fig. 1(a). In addition, by varying the external flux one could prepare the system in a given linear combination of the Andreev states, in close resemblance to the case of a spin-1/2 in a magnetic field. The phase would be, however, affected by fluctuations originated in the coupling of the ring with its electromagnetic environment, characterized by a finite impedance $Z(\omega)$. These fluctuations provide a source of relaxation and dephasing for the dynamics of our two-level system. ${ }^{5}$

The aim of this work is to investigate this dissipative dynamics in order to determine the conditions for the observation of quantum-coherence effects in this system.

For a single mode SQPC in the low bias regime one can neglect the continuous part of the quasiparticle spectrum and restrict the description to the subgap states, introducing the following Hamiltonian, ${ }^{6}$

$$
\hat{H}_{0}=\Delta\left\{\cos \left(\frac{\phi}{2}\right) \hat{\sigma}_{z}+r \sin \left(\frac{\phi}{2}\right) \hat{\sigma}_{x}\right\}
$$

where $\hat{\sigma}_{i}$ are the Pauli matrix and $r=\sqrt{1-\tau}$ is the normal reflection amplitude of the contact. The Hamiltonian adopts the form (2) in the basis of ballistic states and becomes diagonal $\hat{H}_{0}=E_{+}(\phi) \hat{\sigma}_{z}$ in the basis of the Andreev states. The ballistic and the Andreev states are represented in Fig. 1(b).

In the ring geometry, the phase on the contact will be given by $\phi=\phi_{0}-\delta \phi$, where $\phi_{0}=(2 e / \hbar) \Phi$ (being $\Phi$ the external magnetic flux) and $\delta \phi$ represents the phase fluctuations due to the electromagnetic environment. If the impedance viewed by the contact is small compared to the resistance quantum $R_{Q}=h / 4 e^{2}$ one can expand Eq. (2) to the first order in $\delta \phi$ to obtain the coupling between the subgap levels and the environment. Then, the Hamiltonian can be written as $\hat{H}_{0}(\phi)=\hat{H}_{0}\left(\phi_{0}\right)+\hat{H}_{c}$, where the coupling $\hat{H}_{c}$ $=-(\hbar / 2 e) \hat{I} \delta \phi$ is proportional to the current operator $\hat{I}$. Performing a rotation to the basis of the Andreev states, the current operator is given by

$$
\hat{I}=\frac{e \Delta^{2}}{\hbar E_{+}\left(\phi_{0}\right)}\left\{\sqrt{1-\tau} \hat{\sigma}_{x}-\tau \sin \left(\frac{\phi_{0}}{2}\right) \cos \left(\frac{\phi_{0}}{2}\right) \hat{\sigma}_{z}\right\} .
$$

On the other hand, a generic environment can be represented by a set of L-C circuits, with phases $\phi_{n}$, such that $\delta \phi=\Sigma_{n} \phi_{n}$. The relevant information of the environment is contained in the phase-phase correlation function ${ }^{7}$

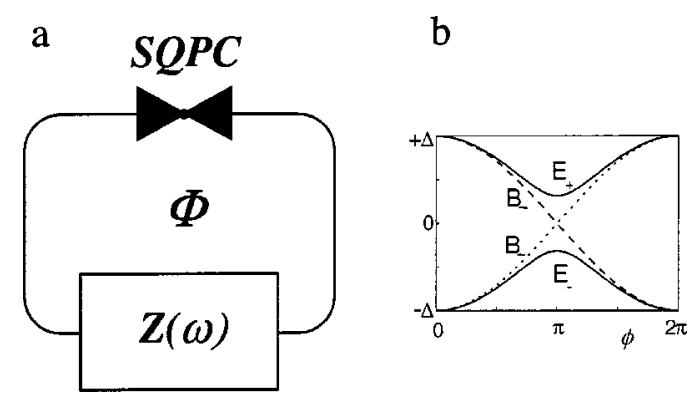

FIG. 1. (a) Schematic circuit: a superconducting quantum point contact (SQPC) inserted in a superconducting ring threaded by a magnetic flux $\Phi . Z(\omega)$ denotes the effective impedance seen by the contact. (b) Andreev levels with phase dependent energies $E_{ \pm}$(full lines). The dotted and dashed lines indicate the ballistic states. 


$$
\begin{aligned}
C(t) & =\sum_{n, m}\left\langle\phi_{n}(t) \phi_{m}(0)\right\rangle \\
& =\frac{1}{R_{Q}} \int_{0}^{\infty} d \omega \frac{\operatorname{Re} Z_{t}(\omega)}{\omega}\left\{\operatorname{coth}\left(\frac{\beta \hbar \omega}{2}\right) \cos \omega t-i \sin \omega t\right\},
\end{aligned}
$$

where $Z_{t}(\omega)$ is the effective impedance of the circuit as seen from the contact and $\beta$ is the inverse temperature.

Projection operator techniques allow to derive a timeconvolutionless generalized master equation ${ }^{8}$ (GME) for the reduced density matrix $\hat{\rho}$ of the Andreev levels. ${ }^{9}$ Up to second order in the coupling Hamiltonian this equation is given by

$$
\begin{aligned}
\dot{\hat{\rho}}(t)+\frac{i}{\hbar}\left[\hat{H}_{0}, \hat{\rho}(t)\right]= & -\frac{1}{\hbar^{2}} \int_{0}^{t} d \tau\{[\hat{I}, \hat{I}(-\tau) \hat{\rho}(t)] C(\tau) \\
& \left.-[\hat{I}, \hat{\rho}(t) \hat{I}(-\tau)] C^{*}(\tau)\right\},
\end{aligned}
$$

where $\hat{I}(\tau)$ denotes the current operator (3) in the interaction representation.

One can extract the evolution equations for the matrix elements of the reduced density projecting the GME (5) in the $\{|+\rangle,|-\rangle\}$ basis of the Andreev states. Taking into account that $\rho_{++}+\rho_{--}=1$, and defining $\rho_{R}$ and $\rho_{I}$ as the real and imaginary part of the off-diagonal matrix element $\rho_{+-}$, we finally get the rate equations

$$
\begin{gathered}
\dot{\rho}_{+}=-\left(W^{+}+W^{-}\right) \rho_{+}+W^{+}-\Lambda \rho_{R}, \\
\dot{\rho}_{R}=\Omega \rho_{I}+\eta \dot{\rho}_{+}, \\
\dot{\rho}_{I}=-\left(W^{+}+W^{-}+\eta \Lambda\right) \rho_{I}-\left(\Omega+2 \Omega_{r}\right) \rho_{R} \\
+\eta \Omega_{r}\left(2 \rho_{+}-1\right)+\Upsilon,
\end{gathered}
$$

where $\rho_{+}=\rho_{++}, \quad \eta=\tau \sin \left(\phi_{0} / 2\right) \cos \left(\phi_{0} / 2\right) / \sqrt{1-\tau}$ and $\Omega$ $=\left(E_{+}-E_{-}\right) / \hbar$.

The coefficients $W^{ \pm}$entering in Eqs. (6) to (8) are the upward and downward transition rates which determine the system relaxation. The dephasing, i.e., the decay of the off diagonal elements, is also controlled by the coefficient $\Lambda$, while $\Omega_{r}$ plays the role of a level shift as discussed below.

The explicit form of all these coefficients depends on the actual impedance $Z_{t}(\omega)$. Typically, this impedance would be determined by the measuring circuit, which could consist of a SQUID inductively coupled to the ring with the SQPC. We shall denote by $M$ and $L$ the mutual inductance between the ring and the SQUID and the self-inductance of the SQUID respectively. The SQUID is also characterized by a finite resistance $R$. The charging effects associated with the contact capacitance can be neglected in the low impedance regime that we are considering. ${ }^{7}$ Under these conditions the real part of $Z_{t}$ will be given by

$$
\operatorname{Re}_{t}(\omega)=\frac{\omega^{2} M^{2} R}{R^{2}+\omega^{2} L^{2}} .
$$

If the environment has a large cuttoff frequency ( $\gamma$ $=R / L \gg \Delta / \hbar)$, we can make the Markov approximation in the evolution equations (6)-(8). In this case, the involved coefficients can be written as

$$
\begin{aligned}
& W^{\mp}=W_{0}^{-}\left\{\begin{array}{l}
n(\Omega)+1, \\
n(\Omega),
\end{array}\right. \\
& \Lambda=W_{0}^{-} \eta \frac{2}{\beta \hbar \Omega}, \\
& \Omega_{r}=W_{0}^{-}\left\{\frac{1}{2} \cot \left(\frac{2}{\beta \hbar \gamma}\right)-\frac{2 \gamma^{2}}{\beta \hbar} \sum_{k=1}^{\infty} \frac{\nu_{k}}{\left[\gamma^{2}-\nu_{k}^{2}\right]\left[\Omega^{2}+\nu_{k}^{2}\right]}\right\}, \\
& \Upsilon=W_{0}^{-} \eta \frac{\Omega}{\gamma}
\end{aligned}
$$

where

$$
W_{0}^{-}=\frac{\pi}{4} \frac{\Delta}{\hbar} \frac{\operatorname{Re} Z_{t}(\Omega)}{R_{Q}} \frac{1-\tau}{\left[1-\tau \sin ^{2}(\phi / 2)\right]^{3 / 2}}
$$

is the downward transition rate at zero temperature, $n(\Omega)$ is the Bose mean occupation number, and $\nu_{k}=2 \pi k / \beta \hbar$ are the Matsubara frequencies.

The actual value of the coefficients is controlled by the environment parameters $M, L$, and $R$. The experimental setup can be designed in order to fix these parameters in the appropriate range to observe quantum-coherence effects. Longer decoherence times would be obtained by reducing $M$ as much as possible. However, SQUID parameters must satisfy certain constraints, imposed by the need to avoid hysteresis and thermal fluctuations. ${ }^{12}$ These considerations permit to estimate a minimal value of $M$ of the order of $0.5 n H$, with $L=0.1 n H$ and $R=20 \Omega .^{13}$ Assuming that the superconducting material is aluminum $\left(\Delta_{A l} \sim 0.18 \mathrm{meV}\right)$, one obtains values of $W_{0}^{-}$in the $n \mathrm{sec}^{-1}$ range. This rate fixes the order of magnitude of the coefficients (10) -(13).

The system of differential equations $(6)-(8)$ is characterized by a set of eigenvalues which determine the typical times for the dissipative dynamics. In the limit of small impedance these eigenvalues are given by the following simple expressions:

$$
\begin{gathered}
\lambda_{1}=-\left(W^{+}+W^{-}\right) \\
\lambda_{2,3}=-\frac{1}{2}\left(W^{+}+W^{-}+2 \eta \Lambda\right) \pm i\left(\Omega+\Omega_{r}\right) .
\end{gathered}
$$

Notice that due to the presence of the environment, the Andreev levels are shifted according to $\widetilde{E}_{ \pm} \simeq E_{ \pm} \pm \Omega_{r} / 2$. However, for the range of parameters we are considering this renormalization is small and can be neglected.

Solving Eqs. (6)-(8) at the lowest order in $\Omega^{-1}$ (which is equivalent to implement the rotating wave approximation ${ }^{14}$ ) one realizes that the eigenvalues (15) are directly connected to the relaxation and dephasing rates by the relations $\Gamma_{R}$ $\simeq-\lambda_{1}$ and $\Gamma_{D} \simeq-\operatorname{Re}\left\{\lambda_{2,3}\right\}$. 
The approximate evolution equations can then be written as

$$
\begin{gathered}
\rho_{+}=\frac{W^{+}}{\Gamma_{R}}\left(1-e^{-\Gamma_{R} t}\right)+\rho_{+}(0) e^{-\Gamma_{R} t}, \\
\rho_{R}=e^{-\Gamma_{D} t}\left[\rho_{R}(0) \cos \Omega t+\rho_{I}(0) \sin \Omega t\right], \\
\rho_{I}=e^{-\Gamma_{D} t}\left[\rho_{I}(0) \cos \Omega t-\rho_{R}(0) \sin \Omega t\right],
\end{gathered}
$$

which clearly shows that the diagonal elements of the density matrix decay exponentially towards their equilibrium values on a time scale given by $1 / \Gamma_{R}$ while the nondiagonal elements perform oscillations with frequency $\Omega$ which are damped on a typical time $1 / \Gamma_{D}$.

This behavior is reflected in the evolution of the current mean value which is given by

$$
\begin{aligned}
\langle I(t)\rangle= & I_{0}\left(1-2 \frac{W^{+}}{\Gamma_{R}}\right)+2 I_{0} e^{-\Gamma_{R} t}\left(\frac{W^{+}}{\Gamma_{R}}-\rho_{+}(0)\right) \\
& +4 e\left(\frac{\Delta}{\hbar}\right)^{2} \frac{\sqrt{1-\tau}}{\Omega} e^{-\Gamma_{D^{t}}\left[\rho_{R}(0) \cos \Omega t\right.} \\
& \left.+\rho_{I}(0) \sin \Omega t\right],
\end{aligned}
$$

where $I_{0}=e(\Delta / \hbar)^{2} \tau \sin \phi / \Omega$ is the equilibrium current at zero temperature.

According to Eq. (19) quantum coherence would manifest as damped Rabi oscillations in the mean current through the contact. These oscillations appear for an initial condition in which both the lower and the upper Andreev state are populated. An arbitrary initial condition can be in principle reached from the equilibrium situation by imposing a given time-dependent evolution to the phase. The amplitude of the oscillations are maximal for $\phi=\pi$ where they become transmission independent. However, by varying the transmission, the frequency of the oscillations could be tuned in order to reach an experimentally accessible range. For instance, in the case of aluminum and transmission $\tau=0.99$ the frequency $\Omega$ would be of the order of $3 \times 10^{10} \mathrm{~Hz}$.

The decay of these oscillations is controlled by the dephasing rate. Figure 2 illustrates the dependence of the relaxation and dephasing rates on the phase $\phi_{0}$, the contact transmission $\tau$ and the temperature. At zero temperature we have $\Gamma_{R}=2 \Gamma_{D}=W_{0}^{-}$, which means that both rates reach a maximum at $\phi_{0}=\pi$ regardless of the contact transmission. This maximum decrease with transmission as $\sqrt{1-\tau}$. At finite but low temperatures we have $\Gamma_{R}=W_{0}^{-}\left(1+2 e^{-\hbar \Omega / k T}\right)$ and $\Gamma_{D}=W_{0}^{-}\left(\frac{1}{2}+2 \eta^{2} k T / \hbar \Omega\right)$. As expected, both rates in-
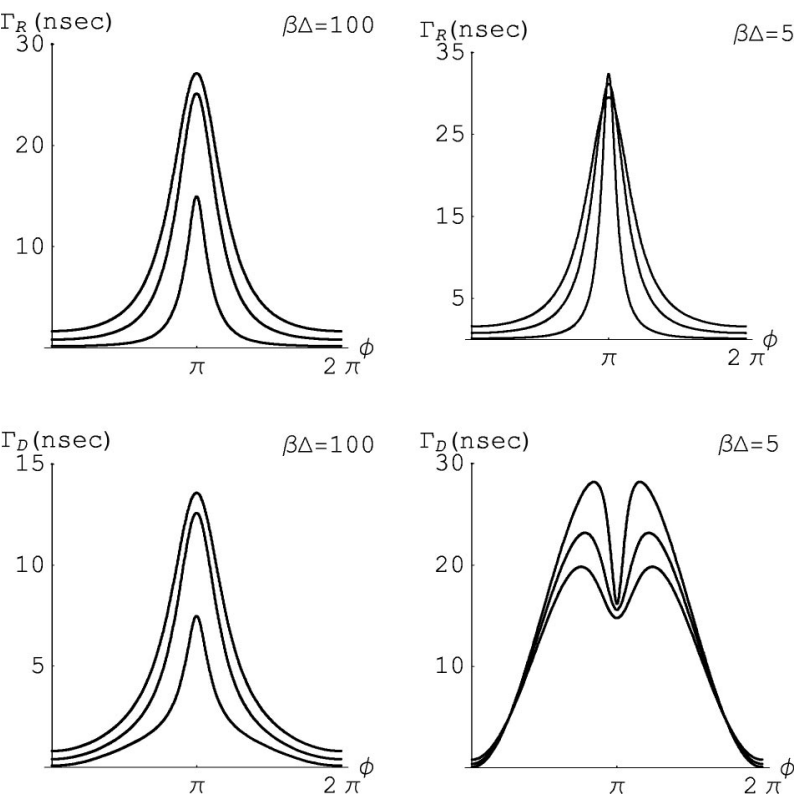

FIG. 2. Relaxation and dephasing rates $\Gamma_{R}$ and $\Gamma_{D}$ as function of the phase $\phi$ for transmission coefficients $\tau=0.9,0.95$, and 0.99 (from top to bottom) and two different values of the parameter $\beta \Delta$. The downward transition rate at zero temperature $W_{0}^{-}$coincides in the graphic scale with $\Gamma_{R}$ for $\beta \Delta=100$.

crease with temperature but the dephasing rate increases linearly instead of exponentially. The maximum of $\Gamma_{R}$ is located at $\phi_{0}=\pi$ for all range of temperatures and behave as $\sqrt{1-\tau} \operatorname{coth} \beta \Delta \sqrt{1-\tau}$. On the other hand, $\Gamma_{D}$ develops a dip at $\phi_{0}=\pi$ and exhibits a double peaked structure located $\phi_{0}=2 \arccos \pm \sqrt{1+[\sqrt{(1-\tau) / 2}-1] / \tau}$ for high temperatures. In this classical limit we get $\Gamma_{R}=W_{0}^{-} 2 k T / \hbar \Omega$ and $\Gamma_{D}=\Gamma_{R}\left(\frac{1}{2}+\eta^{2}\right)$.

In summary, we have studied the dissipative dynamics of the Andreev states in a SQPC coupled to an electromagnetic environment characterized by a generic frequency-dependent impedance. Our results show that with the appropriate choice of the environment parameters one can reach values of the dephasing and relaxation rates within the $n \mathrm{sec}^{-1}$ range. A SQPC in a ring geometry thus provides an interesting realization of a solid-state two-level system in which quantum interference effects could be observed.

We acknowledge useful discussions with C. Urbina, D. Esteve, and A. Martín-Rodero. We are also indebted to F. Vazquez and A. Raguet for their stimulating comments. This work was supported by the Spanish CICyT under Contract No. PB97-0044.
${ }^{1}$ A. Furusaki and M. Tsukada, Physica B 165\&166, 967 (1990); C.W.J. Beenakker, Phys. Rev. Lett. 67, 3836 (1991).

${ }^{2}$ E. Scheer et al., Phys. Rev. Lett. 78, 3535 (1997); E. Scheer et al., Nature (London) 394, 154 (1998).

${ }^{3}$ L.Y. Gorelik et al., Phys. Rev. Lett. 81, 2538 (1998); A. Levy Yeyati, J.C. Cuevas, and A. Martín-Rodero, in Photons and
Local Probes, Vol. 300 of NATO Advanced Study Institute, Series E: Applied Sciences, edited by O. Marti and R. Möller (Kluwer, Dordrecht, 1995).

${ }^{4}$ So far the most advanced results have been obtained on qubits based on flux states in small superconducting loops and on charge states in small superconducting islands. See Y. Nakamura 
et al., Physica B 280, 405 (2000); and V. Bouchiat et al., J. Supercond. 12, 789 (1999).

${ }^{5}$ Other sources of dephasing for Andreev states like Flicker noise have been studied recently by N.I. Lundin and Y.M. Galperin, Phys. Rev. B 63, 094505 (2001).

${ }^{6}$ D.A. Ivanov and M.V. Feigel'man, Phys. Rev. B 59, 8444 (1999).

${ }^{7}$ G.L. Ingold and Yu. V. Nazarov, in Single Charge Tunneling, Vol. 294 of NATO Advanced Study Institute, Series B: Physics, edited by H. Grabert and M.H. Devoret (Plenum, New York, 1991).

${ }^{8}$ T.M. Chang and J.L. Skinner, Physica A 193, 483 (1993).

${ }^{9}$ Notice that our analysis is restricted to the subgap states neglecting coupling with the continuous part of the spectrum. Other interactions, such as electron-phonon coupling, would introduce a broadening of the subgap states (Ref. 10). For aluminum atomic contacts this broadening is, however, small (Ref. 11) and would not introduce important corrections to the relaxation and dephasing rates.

${ }^{10}$ A. Martín-Rodero, A. Levy Yeyati, and F.J. García-Vidal, Phys. Rev. B 53, 8891 (1996); D. Averin and H.T. Imam, Phys. Rev. Lett. 76, 3814 (1996).

${ }^{11}$ M.F. Goffman et al., Phys. Rev. Lett. 85, 170 (2000).

${ }^{12}$ M. Tinkam, Introduction to Superconductivity (McGraw-Hill, New York, 1996).

${ }^{13} \mathrm{C}$. Urbina (private communication).

${ }^{14}$ C. W. Gardiner, Quantum Noise (Springer-Verlag, Heidelberg, 1991). 